\title{
Precise Point Positioning Using the Regional BeiDou Navigation Satellite Constellation
}

\author{
Aigong Xu, Zongqiu Xu, Xinchao Xu, Huizhong Zhu, Xin Sui and \\ Huasheng Sun
}

(School of Geomatics, Liaoning Technical University, Fuxin 123000, China)

(E-Mail: xu_ag@126.com)

On 27 December 2012 it was announced officially that the Chinese Navigation Satellite System BeiDou (BDS) was able to provide operational services over the Asia-Pacific region. The quality of BDS observations was confirmed as comparable with those of GPS, and relative positioning in static and kinematic modes were also demonstrated to be very promising. As Precise Point Positioning (PPP) technology is widely recognized as a method of precise positioning service, especially in real-time, in this contribution we concentrate on the PPP performance using BDS data only. BDS PPP in static, kinematic and simulated real-time kinematic mode is carried out for a regional network with six stations equipped with GPSand BDS-capable receivers, using precise satellite orbits and clocks estimated from a global BDS tracking network. To validate the derived positions and trajectories, they are compared to the daily PPP solution using GPS data. The assessment confirms that the performance of BDS PPP is very comparable with GPS in terms of both convergence time and accuracy.

\section{KEY WORDS}
1. BeiDou Navigation Satellite System.
2. Precise Point Positioning.
3. Real-Time
Positioning.
4. Position Convergence.

Submitted: 19 April 2013. Accepted: 5 December 2013. First published online: 17 January 2014.

1. INTRODUCTION. Since 1985 China has been working on its own navigation satellite system BeiDou (BDS). The system is planned to be established in three steps or phases: demonstrational system, regional system and global system (China National Space Administration, 2003; China Satellite Navigation Office, 2013). On 27 December 2012, the regional system was officially announced to as providing positioning services over the Asia-Pacific region. Now the regional system is in full operation with its designed constellation comprising five Geostationary Earth Orbit (GEO) satellites, five Inclined Geosynchronous Earth Orbit (IGSO) satellites and four Medium Earth Orbit (MEO) satellites. The standard positioning service is free for all civilian users, while the precise positioning service and system-embedded wide area differential positioning are only for authorised users. 
Besides the above-mentioned positioning services, precise relative positioning has already been investigated (Shi et al., 2013; Montenbruck et al., 2012) and precise orbit determination and clock estimation has also been demonstrated (Montenbruck et al., 2012; Ge et al., 2012b; Shi et al., 2012; Steigenberger et al., 2012; Zhao et al., 2013; He et al., 2013). Precise Point Positioning (Zumberge et al., 1997) based on the products has also been undertaken in post-processing and simulated in real-time mode for validating the estimated orbit and clock products (Kouba and Heroux, 2001).

Ge et al. (2012a) carried out BDS PPP in both static and kimematic modes, but only for a single station in the middle of the tracking network. Shi et al. (2012) demonstrated BDS PPP for a single station, which was not involved in orbit and clock estimation. The orbits and clocks were determined with zenith tropospheric delay and receiver clock estimated from GPS data, instead of using BDS as an independent system. In Steigenberger et al. (2012) data from a station in Melbourne, Australia was utilized to test BDS PPP performance in static mode with and without GEOs. Although GEOs have a lower orbit accuracy compared to the IGSOs, they significantly improve the positioning accuracy because of the limited visibility. Zhao et al. (2013) undertook PPP for BDS and BDS + GPS data in both static and kinematic modes. However, again only one station, National Time Service Center (NTSC), China was used and it is several tens of km away from the tracking station $\mathrm{Xi}$ 'An. In general, in most of the studies PPP is carried out for only a single station or a few stations which are not involved in the clock and orbit estimation because of the limited data availability. Real-time kinematic PPP is widely recognized as a mode of precise positioning service, due to its cost-efficiency, global coverage and flexibility (Gao et al., 2003; Bisnath and Gao, 2008). Its performance has been further enhanced through recent developments in PPP ambiguity-fixing (Ge et al., 2008; Laurichesse et al., 2008, Collins et al., 2008) and regional augmentation (Li et al., 2011; Ge et al., 2012a).

The International GNSS Service (IGS) established in 2007 its Real-Time Pilot Project Working Group (http://www.rtigs.net/) and recently announced after about six years of fruitful cooperation its operational real-time service. Thus, BDS PPP is of great interest, especially the capacity of its real-time PPP service. Theoretically, there should not be any significant difference between GPS PPP and BDS PPP thanks to their similar signal structure and analogous frequencies. However, the special BDS constellation with high GEO and IGSO and a few MEO satellites and tracking networks of the BDS regional system will bring some new issues in observation modelling and data processing.

In this contribution, a regional network comprising six stations with an inter-station distance of about $150 \mathrm{~km}$ and equipped with GPS- and BDS-capable dual-frequency receivers were employed for a PPP performance study. Precise orbits and clocks estimated from a global tracking network using the strategy by He et al. (2013) are employed in BDS PPP processing in static, kinematic and simulated real-time kinematic mode. The static results using various session lengths and kinematic trajectories are compared with the daily GPS PPP results using IGS precise orbits and clocks in order to assess the performance of BDS PPP in terms of accuracy and convergence time. After a short description of PPP principles and data processing of the PPP service system, the tracking network and precise products are explained. Then the software package is introduced. The data processing schemata are defined and results are analysed and assessed. Finally, conclusions are drawn accordingly. 
Table 1. Overlapping RMS of the estimated orbits and clocks for each individual satellites and the mean of each satellite type.

\begin{tabular}{|c|c|c|c|c|c|c|}
\hline \multirow{2}{*}{$\frac{\text { Satellite }}{\text { Type }}$} & \multicolumn{4}{|c|}{ Orbit RMS (cm) } & \multicolumn{2}{|c|}{ Clock RMS (ns) } \\
\hline & Along & Cross & Radial & $3 \mathrm{D}$ & STD & RMS \\
\hline GEO & 114 & 10 & 6 & 115 & $0 \cdot 27$ & 0.49 \\
\hline IGSO & 24 & 15 & 7 & 29 & $0 \cdot 31$ & $0 \cdot 37$ \\
\hline MEO & 45 & 13 & 12 & 48 & $0 \cdot 41$ & $0 \cdot 46$ \\
\hline
\end{tabular}

2. EXPERIMENT DESIGN. As is well known, a PPP service system includes two parts: PPP server and client. At the server-end precise orbits and clocks and some additional corrections are generated using data from a reference network and transmitted to the client-end in real-time. At the client-end, most of the biases at the local receiver can be precisely removed by using the products provided by the server. Between the server and client a data link is required for transportation of correction information.

2.1. PPP Server. In this study, the BDS Experimental Test Service (BETS) network with BDS and GPS capability serves as the reference network and was established for the scientific purpose of a Positioning, Navigation and Time (PNT) service by the GNSS Research Center at Wuhan University, China. Since March 2011, 14 stations have been deployed in China and its neighbouring regions. Among them, nine stations are located inside China and five overseas. All stations are equipped with UR240 dual-frequency GPS/BeiDou receivers and UA240 antennas manufactured by the UNICORE Company in Beijing, China (http://www.unicor ecomm.com/english/). A number of studies have been carried out based on data from this network (Ge et al., 2012a; Shi et al., 2012; Zhao et al., 2013; He et al., 2013).

Three-day data are used in a batch estimation to obtain a three-day solution, instead of combining three daily solutions on the level of normal equations (Steigenberger et al., 2011). In order to assess the quality of the estimated clocks and orbits, the differences over the overlapping time of two adjacent three-day solutions are utilized as usual. The orbit of the last day in a three-day solution is compared with that of the middle day of the next three-day solution. The RMS of the differences in along-track, cross-track and radial directions are taken as an orbit quality indicator. The statistical results for orbits and clocks are listed in Table 1.

From the orbit RMS in Table 1, the along-track RMS is significantly larger than that of the other two directions as expected. GEOs have the largest RMS in the alongtrack direction of $114 \mathrm{~cm}$, compared to $24 \mathrm{~cm}$ and $45 \mathrm{~cm}$ for IGSOs and MEOs, respectively. RMS in cross-track and radial are very similar for the three types of satellites, i.e., $10 \mathrm{~cm}$ and $6 \mathrm{~cm}$, respectively, for GEOs, $15 \mathrm{~cm}$ and $7 \mathrm{~cm}$, respectively, for IGSOs, and $13 \mathrm{~cm}$ and $12 \mathrm{~cm}$, respectively, for MEOs.

2.2. PPP Client. Aside from the above mentioned network, a regional network comprising six different stations (Xu et al., 2013) equipped with the same UNICORE receivers and antenna were also employed as user stations for the tests of various precise point positioning modes. The inter-station distance is about $150 \mathrm{~km}$ on average. The distribution of the stations and their locations with respect to the BETS network are shown in Figure 1. 


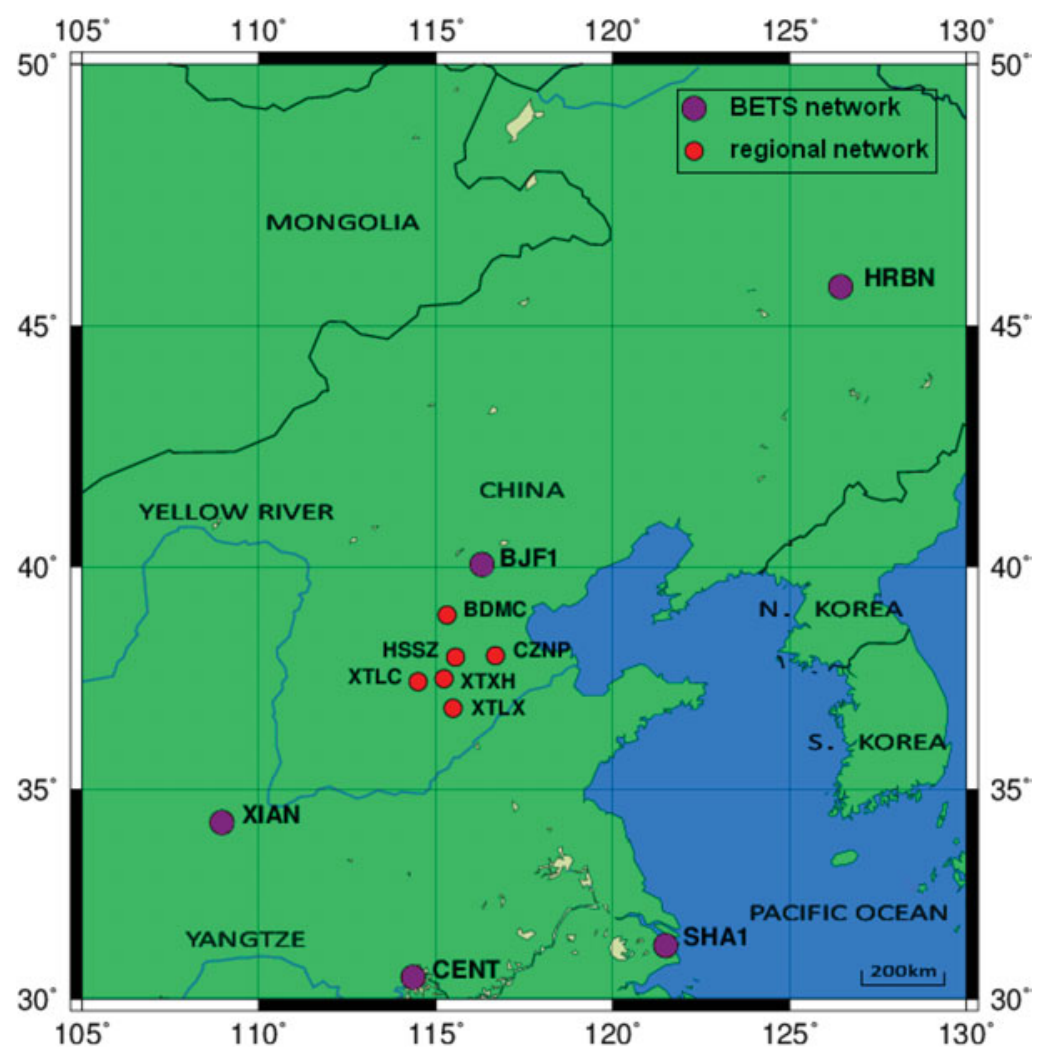

Figure 1. The regional BDS/GPS test network within the context of the global BDS reference network in China.

\section{DATA PROCESSING}

3.1. Software package. The PANDA (Positioning And Navigation Data Analyst) software is used for the data processing. The software was developed at the GNSS Research Center in Wuhan University (Liu and Ge, 2003; Shi et al., 2008) and has recently been adapted for BDS data processing (Shi et al., 2012; Zhao et al., 2013; He et al., 2013). Similar to other scientific GNSS software packages, it includes the following basic modules: data pre-processing, orbit integration, parameter estimation, data editing and ambiguity-fixing. Two estimators have been developed: a least square estimator for post-mission processing and a square root information filter estimator for real-time processing.

3.2. Models. The undifferenced ionosphere-free code and phase observations are employed in the PPP processing. The a priori STD of B1 and B2 phase and range are 0.01 cycles and $1.0 \mathrm{~m}$, respectively. Observations are down-weighted by factor of $2^{*} \sin (\mathrm{E})$ if their elevations are lower than $30^{\circ}$ and are excluded if their elevations are below $7^{\circ}$. Satellite orbits and clocks and Earth rotation parameters are fixed to the products estimated from the BETS network at the server-end. The relativistic delay and station displacement caused by solid earth tides, pole tides and ocean tide loading are corrected following the instruction or using the models and subroutines 
recommended in IERS Convention 2003. The phase windup is calculated and corrected using the formulae in Wu et al. (1993).

Tropospheric delays are first corrected through ZTD computed using the Saastamoinen model and meteorological data from the Global Pressure and Temperature model (GPT) (Boehm et al., 2007), as well as the Vienna Mapping Function (VMF) (Boehm et al., 2006). The remaining ZTD are modeled by a randomwalk stochastic process with a power density of $2.5 \mathrm{~mm} / \sqrt{h}$ (Bar-Sever and Kroger, 1998). Receiver clock error is estimated as white noise. Ambiguities are estimated as real-values and integer ambiguity resolution is not performed. Station coordinates are estimated for static mode as determined parameters with a loose constraint of about $100 \mathrm{~m}$ for each component and for kinematic mode as a first GaussianMarkov process with $100 \mathrm{~m}$ constraint on initial coordinates and a power density of about $1 \mathrm{~m} / \mathrm{s}$.

Phase Centre Offset (PCO) and Phase Centre Variation (PCV) of BDS satellites and receivers are not currently available. Therefore, PCO and PCV corrections are neglected. Satellite attitude control mode has also not been announced. Moreover, the attitude control mode of the BDS satellites is assumed to be the same as that of the GPS Block IIR satellites (He et al., 2013).

3.3. Processing Schemata. Three processing scenarios are defined for assessing the performance of the potential PPP services: static mode with various lengths of observations, kinematic positioning in post-mission mode, and simulated real-time kinematic positioning.

In static mode, data from the three selected days are divided into sessions of various lengths; estimated station coordinates are compared with the multi-day GPS solution; and their repeatability is also utilized as a quality indicator for their internal consistency. We used session lengths of $2 \mathrm{~h}, 3 \mathrm{~h}, 6 \mathrm{~h}, 12 \mathrm{~h}$ and $24 \mathrm{~h}$.

Kinematic solutions with daily data are carried out in post-processing mode. The station coordinates are modelled by a first order Gaussian-Markov process. In the least squares adjustment, the state equation of the process is added as pseudo observation equations into the normal equation system. Station coordinates at the previous epochs are eliminated and they are recovered backwards after the parameters at the last epoch are solved. Therefore the estimated coordinates are equivalent to those from a total least squares adjustment using all of the observations - a smoothed solution. We tried to investigate the capability of the BDS real-time PPP service but in simulated real-time mode.

We used estimates of a three-day solution of precise orbit determination as the basis to predict the orbits of the day after. With the predicted orbits as known values, satellite clocks of the next day were estimated epoch-by-epoch with fixed station coordinates. Simulated real-time positioning using the estimated orbits and clocks was performed to validate the accuracy of BDS real-time positioning service.

As is well known, the convergence time of real-time kinematic PPP is a key indicator of its performance. In order to estimate the convergence time, we divided the data into two-hour sessions and each is processed in simulated kinematic mode. For each station there are 12 solutions every day and 216 solutions in total for six stations over three days. The estimated trajectories are compared with the known coordinates. The RMS of the coordinate differences at the same epoch number of all the solutions are computed to illustrate the relationship between length of observations and position accuracy. 


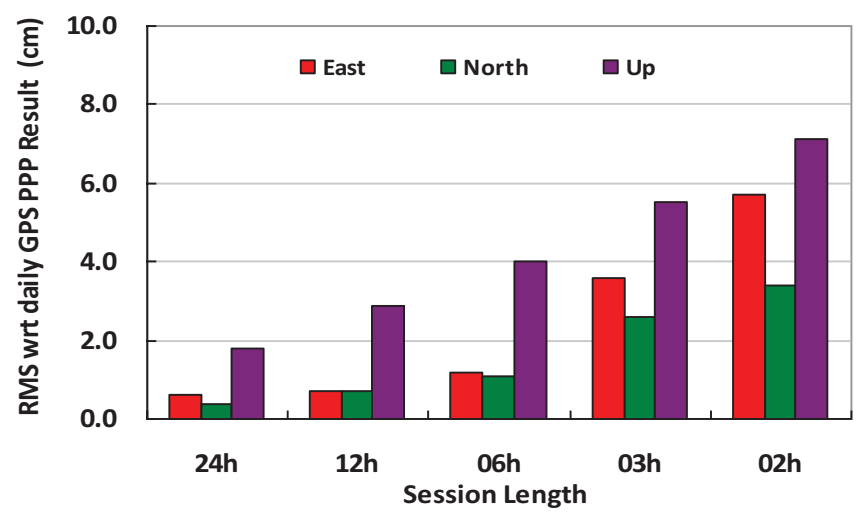

Figure 2. RMS of the positioning differences in east, north and up directions of the static PPP with various session lengths over the three selected days.

4. RESULTS. Data from days 311-313 of 2012 of the six regional stations are selected as user stations in the three processing scenarios defined above. The estimated station coordinates are compared with those estimated from GPS data using IGS final products. The details are discussed in the following.

4.1. Static PPP. In the static test, PPP is performed with session lengths of two hours, three hours, six hours, 12 hours and 24 hours. Figure 2 shows the averaged RMS of the coordinate differences in east, north and up directions of the static solutions with various session lengths over the three selected days.

The accuracy of the PPP derived positions with two hours data is about $6 \mathrm{~cm}, 4 \mathrm{~cm}$ and $7 \mathrm{~cm}$ in east, north and up directions, respectively, compared to the GPS daily solution. The accuracy is improved along with the session lengths. If data of six hours or longer are involved in the processing, position accuracy in the east and north is about $1 \mathrm{~cm}$, while there is a bias of about $3 \mathrm{~cm}$ in the up direction.

Figure 3 shows the time series of the differences for station HSSZ as an example. The three panels are for days 311, 312 and 313 from top to bottom, respectively. In each panel, all solutions of the $3 \mathrm{~h}, 6 \mathrm{~h}, 12 \mathrm{~h}$ and $24 \mathrm{~h}$ sessions are presented from left to right. The solution of the $3 \mathrm{~h}$ sessions is rather noisy and there is clearly a systematic bias in the vertical component. The bias in the vertical is most likely caused by the unmodelled PCO and PCV of the receiver and satellite antennas as well. Hence, vertical bias is present in all arc lengths.

4.2. Kinematic PPP. Kinematic PPP is also applied to the regional stations using the same orbit and clock products as for static PPP for the days 311-313 in 2012. The positions are estimated by means of the sequential least square adjustment with a loose constraint of $1 \mathrm{~m}$ to positions at two adjacent epochs. The epoch-wise station coordinates are eliminated at each epoch while processing forwards and are recovered backwards (Ge et al., 2006).

The derived trajectories are compared to the daily PPP solution using GPS data. The bias and STD of the differences in east, north and up are shown in Figure 4 for the six stations. Both bias and STD are below $10 \mathrm{~mm}$ for east component, $10 \mathrm{~mm}$ and $15 \mathrm{~mm}$ for north component, respectively, about $-40 \mathrm{~mm}$ and $50 \mathrm{~mm}$ for vertical component, respectively. 

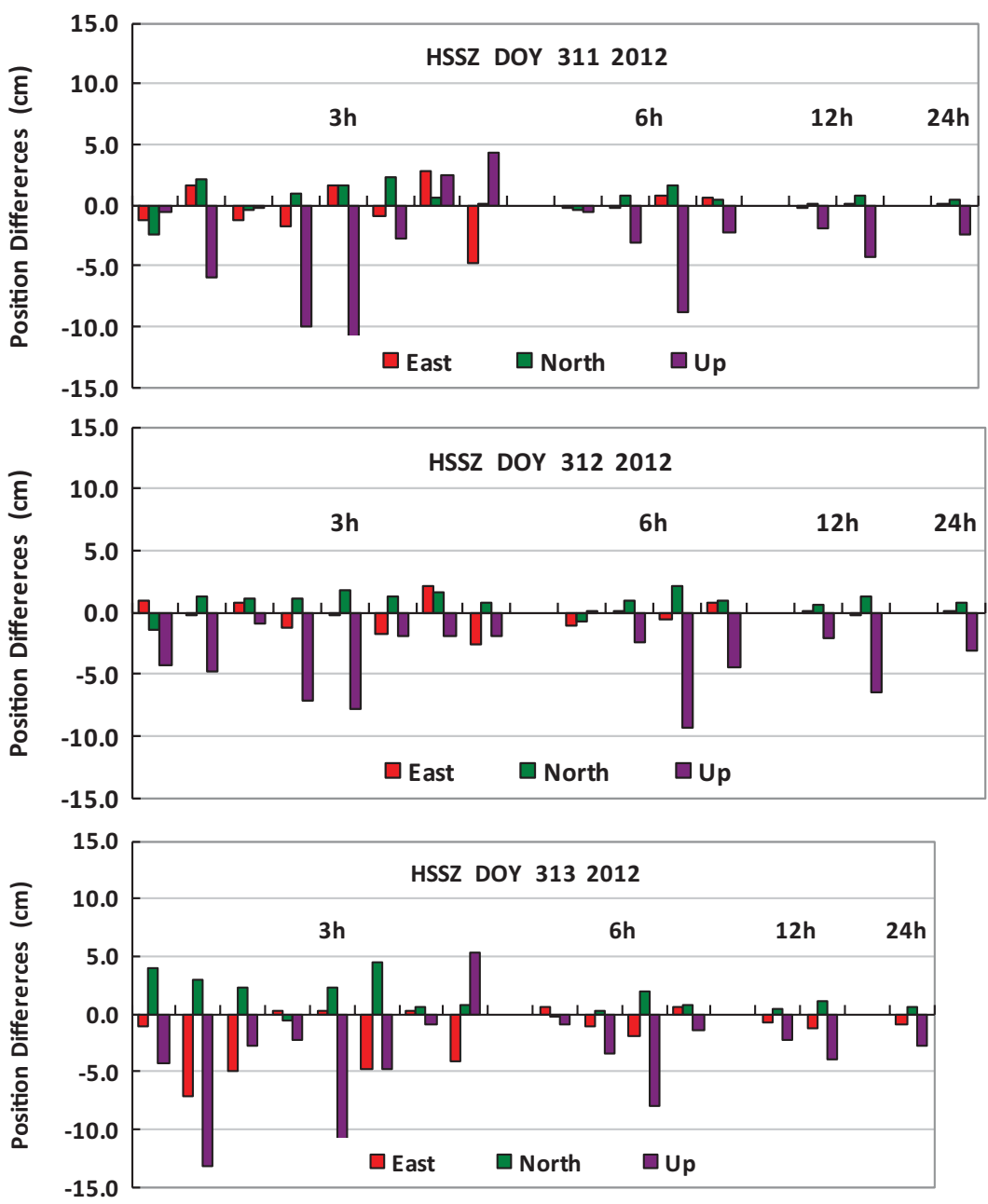

Figure 3. Position differences of static PPP solutions with session length of 3 hours, 6 hours, 12 hours and 24 hours for days 311, 312 and 313 compared to the estimates using daily GPS data for station HSSZ.

As an example, position differences of the trajectory for the six stations on day of year (DOY) 312 in 2012 are displayed in Figure 5 and the number of satellites used in each epoch is also shown. There is an obvious variation pattern in the vertical time series that differs from station to station. The reason is still under investigation and it could potentially be the multi-path effect or any inaccurate modelling.

4.3. Simulated Real-Time PPP service. Figure 6 presents the position differences of the simulated real-time PPP service and the static daily GPS solution. The number of observed satellites is also plotted. Compared to the real-time PPP result in Figure 6 and the post-processing result in Figure 5, a long convergence time of about $1 \cdot 5$ hours is needed for real-time PPP in order to get positions of about $10 \mathrm{~cm}$ accuracy. Afterwards, the accuracy stays at the sub-dm-level. 

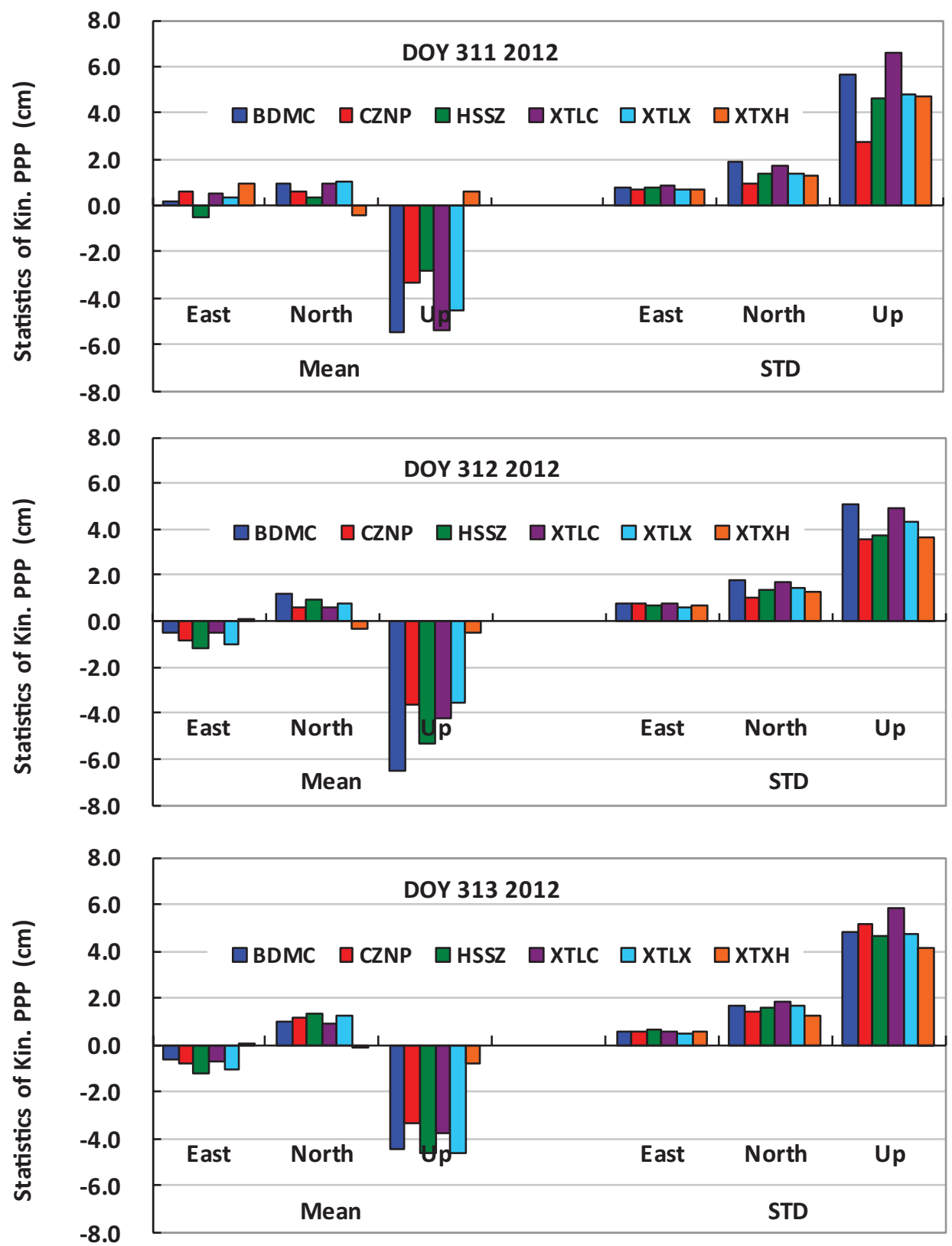

Figure 4. Bias (left) and STD (right) of the derived trajectories of the six regional stations in kinematic mode.

According to the processing schema, for each epoch number, there are 216 estimated coordinates. Their differences with respect to the known coordinates reflect the position quality with the data up to this epoch. Therefore, the RMS of all the 216 coordinate differences can be a measure of the position accuracy. Figure 7 shows the relationship between the length of observations and the accuracy of the 
BDMC DOY 3122012

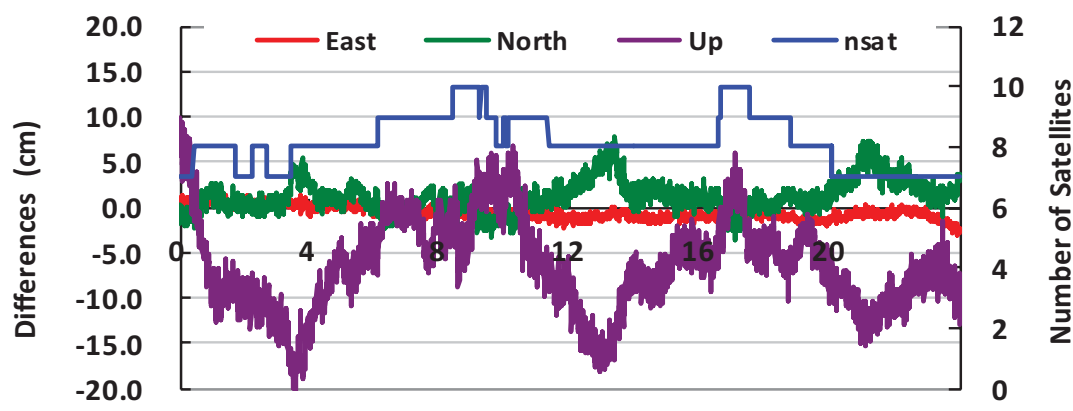

CZNP DOY 3122012

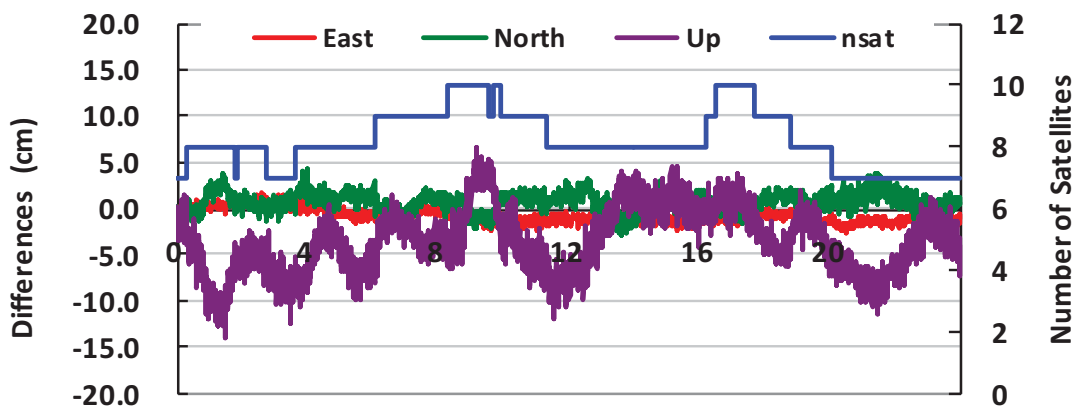

HSSZ DOY 3122012
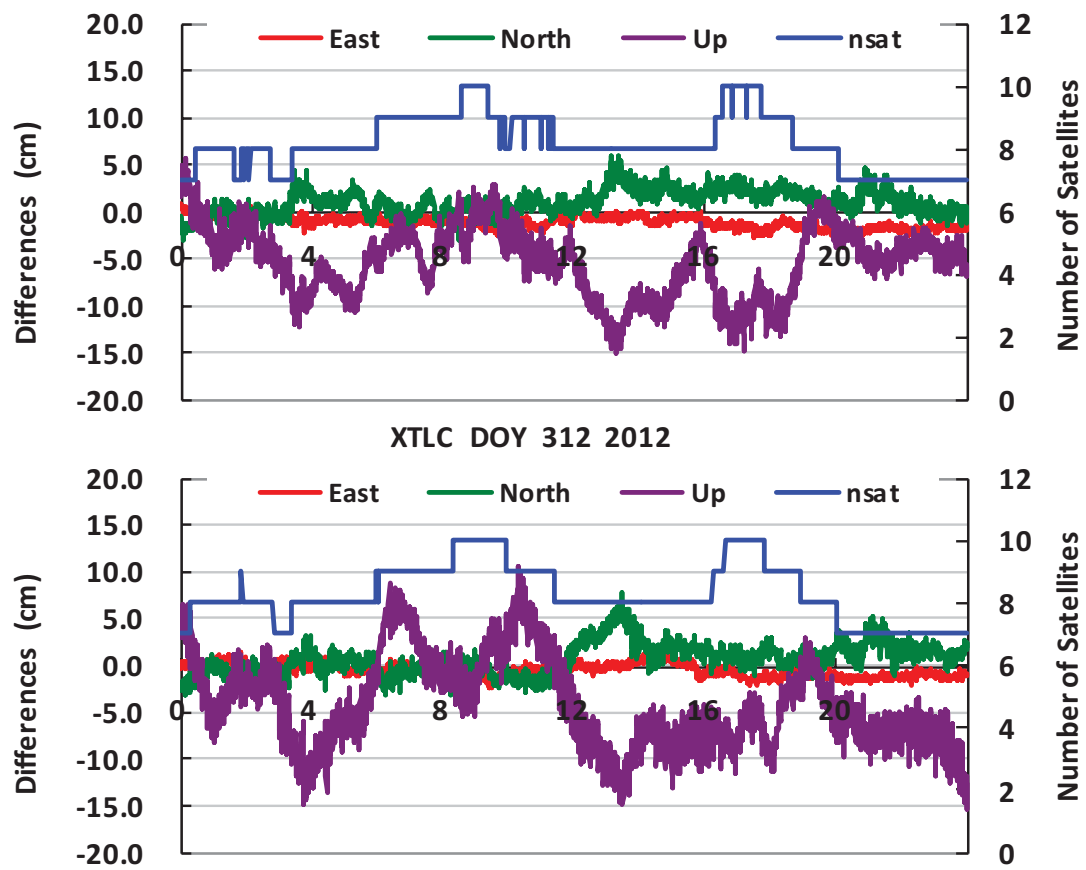

Figure 5. For legend see next page. 
XTLX DOY 3122012

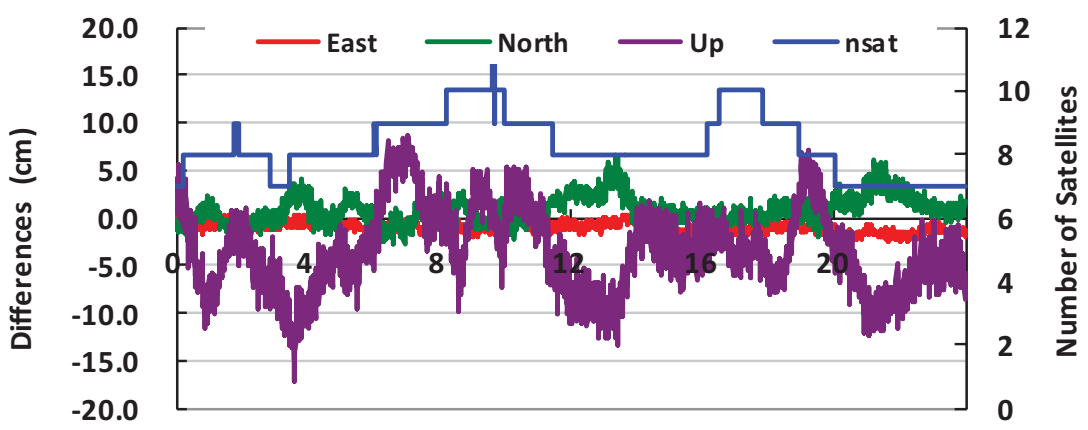

XTXH DOY 3122012

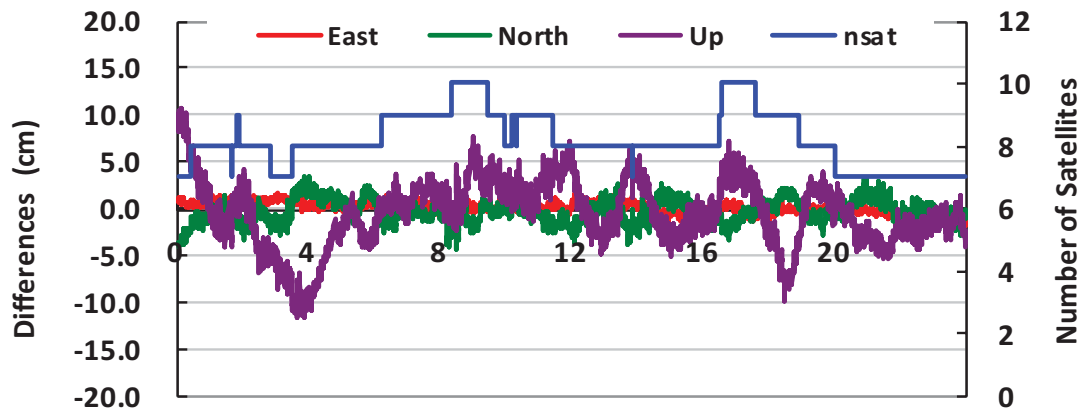

Figure 5. Position differences of the kinematic PPP and number of satellites observed for the six regional stations on DOY 312 in 2012.

estimated positions. From Figure 7, we have similar accuracy for the east and up components although usually the east should be much better than the up component. After one hour convergence, the accuracy is about $8 \mathrm{~cm}, 20 \mathrm{~cm}$ and $20 \mathrm{~cm}$ for the north, east and up directions, respectively. With 90 minutes data, horizontal accuracy is better than $10 \mathrm{~cm}$ on average in the north and east components and about $15 \mathrm{~cm}$ for the vertical. The accuracy is still improved along with observing time and reaches to better than $10 \mathrm{~cm}$ for all components at the end of the two-hour session.

The bias and STD of the differences in simulated real-time PPP after 1.5 hours are shown on Figure 8 for the six stations. The bias is below $10 \mathrm{~mm}$ for east and north components, about $-30 \mathrm{~mm}$ for the vertical component. The STD is about $20 \mathrm{~mm}$ for east and north components, about $60 \mathrm{~mm}$ for the vertical component. Therefore, with the BDS regional system, positioning accuracy of better than one decimetre is achievable in real-time PPP mode.

5. SUMMARY. The BDS navigation satellite system being established in China is moving steadily forward towards its final destination - an operational global navigation satellite system by 2020 . The three-phase schedule enables the rapid emerging of the BDS system with its operational service over the Asia-pacific region now. 
BDMC DOY 3112012
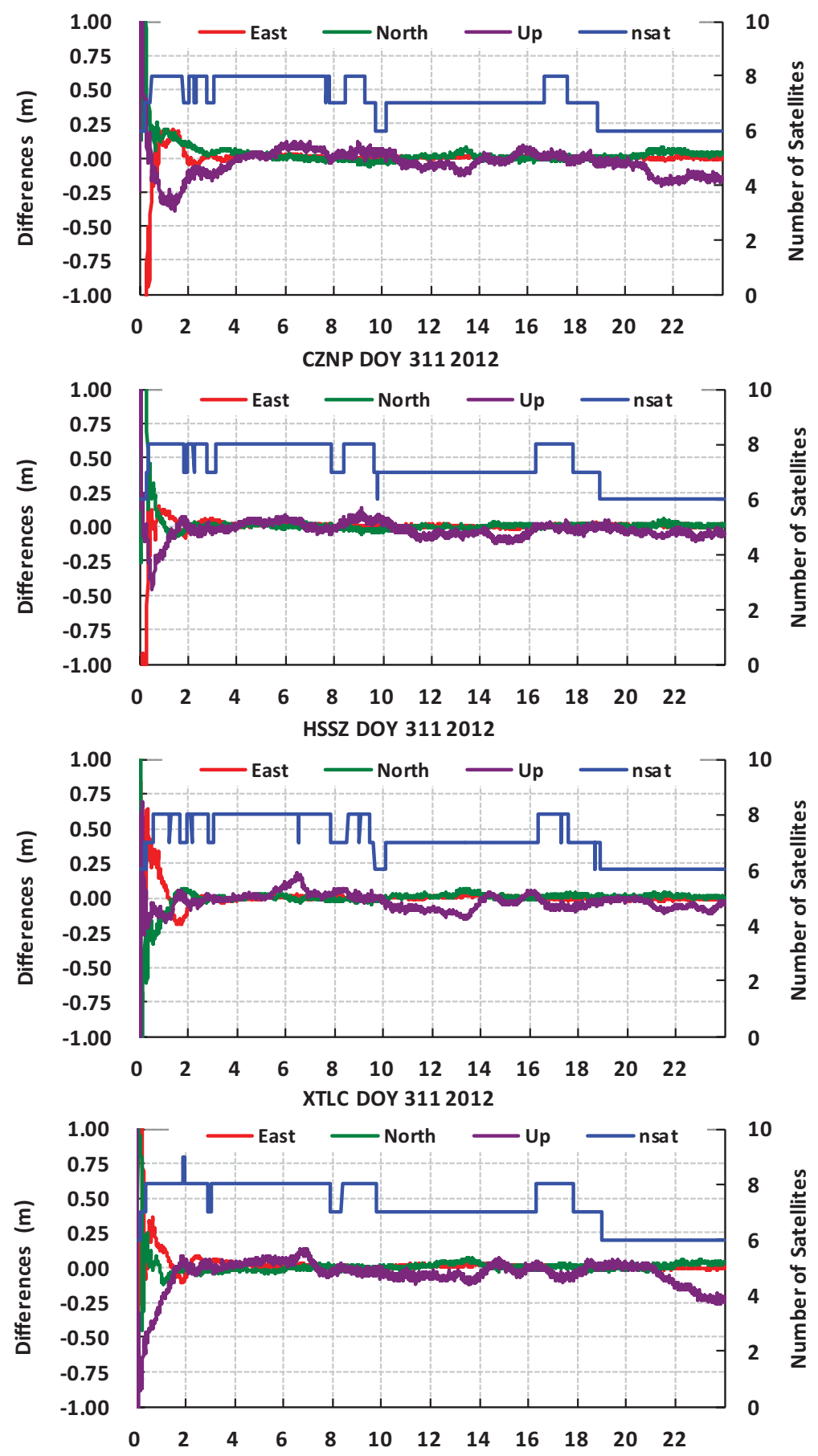

Figure 6. For legend see next page. 

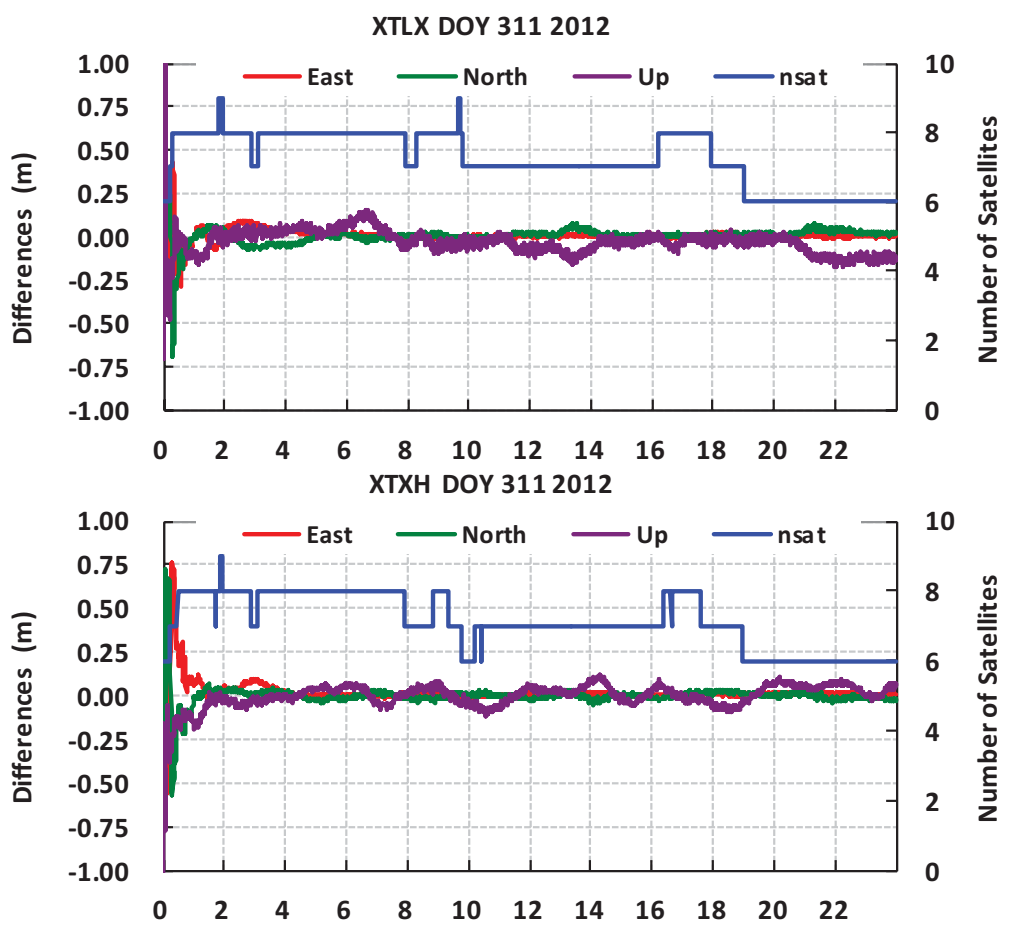

Figure 6. Position differences of the simulated real-time and the static daily PPP for the six stations on DOY 311 in 2012.

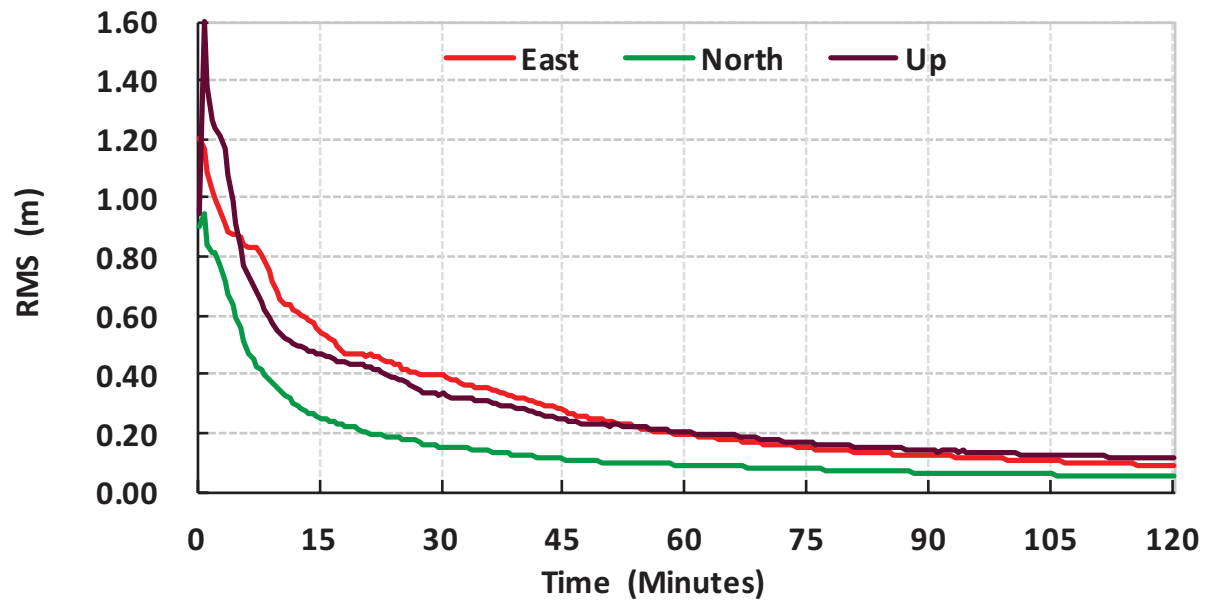

Figure 7. Convergence of station position along with the length of observations.

With the precise orbit and clock estimated from the BETS tracking network based on Chinese stations and several stations worldwide, PPP in static, kinematic and simulated real-time modes using BDS data only have been carried out and assessed. 


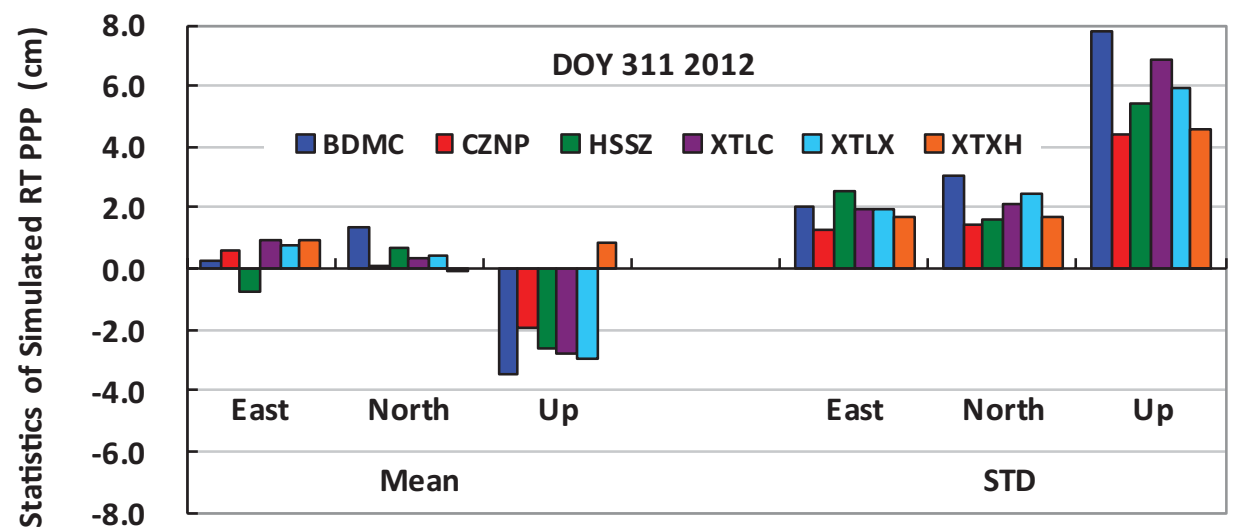

Figure. 8 Bias (left) and STD (right) of the derived trajectories of the six stations in simulated realtime mode after 1.5 hours.

In the static test, an accuracy of a few $\mathrm{cm}$ is attainable with about two-hour data compared to the GPS daily PPP solution. The accuracy in terms of RMS increases along with the observation time and reaches to better than $1 \mathrm{~cm}$ in the horizontal and $4 \mathrm{~cm}$ in the vertical with six hours data or longer. A clear bias changing along with observing time is found in the vertical direction, potentially being caused by the un-modelled PCO and PCV of the receiver and satellite antennas.

For kinematic post-positioning, the horizontal accuracy in STD is about 1 to $2 \mathrm{~cm}$, but about $5 \mathrm{~cm}$ for vertical but with a bias of $-4 \mathrm{~cm}$ which is about $6 \mathrm{~cm}$ in RMS. There are clearly long-term variations in the position time series, especially in the vertical direction, compared to the ground truth.

Simulated real-time PPP shows that after a convergence time of about 60 minutes, position accuracy reaches to $10 \mathrm{~cm}, 20 \mathrm{~cm}$ and $20 \mathrm{~cm}$ in north, east and up, respectively. On average, a convergence time of 90 minutes is needed for obtaining positions with an accuracy of about $10 \mathrm{~cm}$. The accuracy of the converged simulated real-time kinematic positioning is 2 to $3 \mathrm{~cm}$ in the horizontal and 6 to $7 \mathrm{~cm}$ in the vertical.

Therefore, the operational BDS system can provide very precise positioning services similar to GPS and GLONASS, but only to China and its surroundings. Although the performance in terms of convergence time and accuracy is still slightly worse than GPS, these metrics can potentially be improved by applying accurate satellite and receiver PCO and PCV corrections and satellite attitude information.

\section{ACKNOWLEDGEMENTS}

The authors would like to thank the GNSS Research Center at Wuhan University and the Chinese Academy for Surveying and Mapping for providing the BDS data of the BETS network and the regional network for this study. This research is supported by the Liaoning Province Excellent Talent Program (LR201018), the PhD Programs Foundation of MOE of China (2012212111007), and the National Natural Science Foundation of China (41201454). 


\section{REFERENCES}

Bar-Sever, Y. E. and Kroger, P. M. (1998). Estimating Horizontal Gradients of Tropospheric Path Delay with a Single GPS Receiver. Journal of Geophysical Research. 103 (B3), 5019-5035.

Boehm, J., Werl, B. and Schuh, H. (2006). Troposphere mapping functions for GPS and very long baseline interferometry from European Centre for Medium-Range Weather Forecasts operational analysis data. Journal of Geophysical Research, 111, B02406. doi: 10.1029/2005JB003629.

Boehm, J., Heinkelmann, R. and Schuh, H. (2007). Short Note: A global model of pressure and temperature for geodetic applications. Journal of Geodesy, 81, 679-683.

Bisnath, S. and Gao, Y. (2008). Current State of Precise Point Positioning and Future Prospects and Limitations. International Association of Geodesy Symposia, 133, 615-623.

China National Space Administration (2003). Comparable with American and Russian in terms of performance, BeiDou-1 navigates for China, (in Chinese), 30 May 2003. Retrieved 19 May 2010.

China Satellite Navigation Office (2013). BeiDou Navigation Satellite System Signal in Space Interface Control Document. Available online: http://gge.unb.ca/test/beidou_icd_english.pdf (accessed on 1 April 2013).

Collins, P., Lahaye, F., Héroux, P. and Bisnath, S. (2008) Precise point positioning with ambiguity resolution using the decoupled clock model. Proceedings of ION GNSS 2008, Sept. 16-19, Savannah, GA, USA.

Gao, Y., Chen, K. and Shen, X. (2003). Real-time Kinematic Positioning Based on Un-differenced Carrier Phase Data Processing. Proceedings of ION National Technical Meeting, Anaheim, California, January 22-24, 2003.

Ge, M., Gendt, G., Dick, G., Zhang, F. and Rothacher, M. (2006). A new data processing strategy for huge GNSS global networks. Journal of Geodesy, 80(4), 199-203.

Ge, M., Gendt, G., Rothacher, M., Shi, C. and Liu, J. (2008). Resolution of GPS carrier-phase ambiguities in Precise Point Positioning (PPP) with daily observations. Journal of Geodesy, 82, 389-399.

Ge, M., Dousa, J., Li, X., Ramatschi, M., Nischan, T. and Wickert, J. (2012a). A Novel Real-Time Precise Positioning Service System: Global Precise Point Positioning with Regional Augmentation. Journal of Global Positioning System, 11(1), 2-10. doi: 10.5081/jgps.11.1.2.

Ge, M., Zhang, H. P., Jia, X. L., Song, S. L. and Wickert, J. (2012b). What Is Achievable with the Current COMPASS Constellation? GPS World, November, 29-34.

He, L., Ge, M., Wang, J., Wickert, J. and Schuh, H. (2013). Experimental Study on the Precise Orbit Determination of the BeiDou Navigation Satellite System. Sensors, 13, 2911-2928.

Kouba, J. and Heroux, P. (2001). Precise Point Positioning Using IGS Orbit and Clock Products. GPS Solutions, 5(2), 12-28.

Laurichesse, D., Mercier, F., Berthias, J., Broca, P. and Cerri, L. (2008). Zero-difference Ambiguity Fixing for Spaceborne GPS Receivers, Proceedings of ION GNSS 2008, Savannah, Georgia, September 16-19, 2008, 758-768.

Li, X., Zhang, X. and Ge, M. (2011). Regional reference network augmented precise point positioning for instantaneous ambiguity resolution. Journal of Geodesy, 85(3): 151-158. doi: 10.1007/s00190-0100424-0.

Liu, J. and Ge, M. (2003). PANDA software and its preliminary result of positioning and orbit determination. Wuhan University Journal of Natural Sciences, 8, 603-609.

Montenbruck, O., Hauschild, A., Steigenberger, P., Hugentobler, U., Teunissen, P. and Nakamura, S. (2012). Initial assessment of the COMPASS/BeiDou-2 regional navigation satellite system. GPS Solutions. doi: 10.1007/s10291-012-0272-x.

Shi, C., Zhao, Q., Geng, J., Lou, Y., Ge, M. and Liu, J. (2008). Recent Development of PANDA Software in GNSS Data Processing. In Proceedings of the Society of Photographic Instrumentation Engineers 7285, International Conference on Earth Observation Data Processing and Analysis (ICEODPA), 72851S, Wuhan, China, 28 December 2008. doi: 10.1117/12.816261.

Shi, C., Zhao, Q., Li, M., Tang, W., Hu, Z., Lou, Y., Zhang, H., Niu, X. and Liu, J. (2012). Precise orbit determination of BeiDou Satellites with precise positioning. Science China Earth Sciences, 55, 1079-1086.

Shi, C., Zhao, Q., Hu, Z. and Liu, J. (2013). Precise relative positioning using real tracking data from COMPASS GEO and IGSO satellites. GPS Solutions, 17, 103-119.

Steigenberger, P., Hugentobler, U., Montenbruck, O. and Hauschild, A. (2011). Precise orbit determination of GIOVE-B based on the CONGO network. Journal of Geodesy, 85, 357-365. 
Steigenberger, P., Hauschild, A., Montenbruck, O. and Hugentobler, U. (2012). Performance Analysis of COMPASS Orbit and Clock Determination and COMPASS-Only PPP. In IGS Workshop, Olsztyn, Poland, 23-27 July, 2012.

Wu, J.T., Wu, S.C., Hajj, G.A., Bertiger, W.I. and Lichten, S.M. (1993). Effects of antenna orientation on GPS carrier phase. Manuscr. Geod, 18, 91-98.

Xu, A., Xu, Z., Ge, M., Xu, X., Zhu, H. and Sui, X. (2013). Estimating Zenith Tropospheric Delays From BeiDou Navigation Satellite System Observations. Sensors, 13(4), 4514-4526; doi:10.3390/s130404514.

Zhao, Q., Guo, J., Li, M., Qu, L., Hu, Z., Shi, C. and Liu, J. (2013). Initial results of precise orbit and clock determination for COMPASS navigation satellite system. Journal of Geodesy, doi: 10.1007/s00190-0130622-7.

Zumberge, J.F., Heflin, M.B., Jefferson, D.C., Watkins, M.M. and Webb, F.H. (1997). Precise point positioning for the efficient and robust analysis of GPS data from large networks. Journal of Geophysical Research, 102(B3), 5005-5017. 